\title{
L1.1 Is Involved in Spinal Cord Regeneration in Adult Zebrafish
}

\author{
Catherina G. Becker, Bettina C. Lieberoth, Fabio Morellini, Julia Feldner, Thomas Becker, and Melitta Schachner \\ Zentrum für Molekulare Neurobiologie, Universität Hamburg, D-20246 Hamburg, Germany
}

\begin{abstract}
Adult zebrafish, in contrast to mammals, regrow axons descending from the brainstem after spinal cord transection. L1.1, a homolog of the mammalian recognition molecule L1, is upregulated by brainstem neurons during axon regrowth. However, its functional relevance for regeneration is unclear. Here, we show with a novel morpholino-based approach that reducing L1.1 protein expression leads to impaired locomotor recovery as well as reduced regrowth and synapse formation of axons of supraspinal origin after spinal cord transection. This indicates that L1.1 contributes to successful regrowth of axons from the brainstem and locomotor recovery after spinal cord transection in adult zebrafish.
\end{abstract}

Key words: regeneration; cell recognition molecule; spinal cord; brainstem; teleost; Danio rerio

\section{Introduction}

Axotomized neurons regenerating an axon show increased expression of so-called growth-associated genes, which may have important functions for axon regrowth (for review, see Bulsara et al., 2002; Plunet et al., 2002). One of these genes is L1, a homophilically and heterophilically binding cell surface protein of the immunoglobulin superfamily of cell recognition molecules that is involved in many aspects of nervous system development, including axon growth and synaptic plasticity (for review, see Haspel and Grumet, 2003). During peripheral nerve regeneration, L1 is expressed in motor neurons and Schwann cells (Martini and Schachner, 1988; Zhang et al., 2000). In the mammalian CNS, expression of L1 is increased in subpopulations of axotomized neurons only when their axons regrow into a permissive peripheral nerve graft (Zhang et al., 1995). Infusion of L1-Fc fusion protein into the lesioned adult rat spinal cord promotes locomotor recovery (Roonprapunt et al., 2003). These findings indicate that increased expression of L1 may be relevant for axon regrowth to occur.

In zebrafish, many axons, including those that project from the brainstem to the spinal cord, regrow in the adult CNS after a lesion, and fish spontaneously recover locomotor aptitude after spinal lesion within several weeks (Becker et al., 1997). There are two L1 homologs in zebrafish, designated L1.1 and L1.2 (Tongiorgi et al., 1995). As in mammals, increased expression of these genes is tightly correlated with regenerative success of CNS axons such as retinal ganglion cells (Bernhardt et al., 1996) or brainstem

Received May 5, 2004; revised July 26, 2004; accepted July 27, 2004.

This work was supported by the Christopher Reeve Paralysis Foundation (C.G.B., T.B.), the Deutsche Forschungsgemeinschaft Grant Be 1650/3-3 (T.B., M.S.), and the University Hospital Eppendorf special fund for promotion of women in science (M.S.). We thank Linda Schuldt for excellent technical assistance and Dr. Alexander G. Nikonenko for advice on statistical analysis.

Correspondence should be addressed to Dr. Catherina G. Becker, Zentrum für Molekulare Neurobiologie, Universität Hamburg, Martinistrasse 52, D-20246 Hamburg, Germany. E-mail: tcbecker@zmnh.uni-hamburg.de. DOI:10.1523/JNEUROSCI.2420-04.2004

Copyright $\odot 2004$ Society for Neuroscience $\quad 0270-6474 / 04 / 247837-06 \$ 15.00 / 0$ neurons with spinal axons (Becker et al., 1998). Of the two homologs, L1.1 is most prominently upregulated after spinal cord lesion. A detailed analysis of L1.1 expression in the brainstem shows that the proportion of neurons in different brainstem nuclei that upregulate expression of L1.1 correlates with differences in regenerative capacity of these nuclei (our unpublished observations; Becker et al., 1998).

To show directly that expression of L1.1 is a necessary component of the regenerative response of brainstem neurons in adult zebrafish, we used antisense morpholino oligonucleotides (Nasevicius and Ekker, 2000; Heasman, 2002) to suppress expression of L1.1 during regeneration. We were able to efficiently apply morpholinos by retrograde transport into the somata of axotomized neurons in the brainstem of adult zebrafish, which leads to reduced L1.1 protein expression in these neurons. Using retrograde axonal tracing and a behavioral test of locomotion, we found that regrowth of axons from the brainstem and locomotor recovery are both strongly impaired by the knockdown of L1.1, indicating that this molecule is an important player in spinal cord regeneration of a vertebrate.

\section{Materials and Methods}

Spinal cord lesion and application of tracers and morpholinos. All of the animal experiments were approved by the University and State of Hamburg animal care committees. For regeneration experiments, spinal cords were lesioned as described previously (Becker et al., 1997). Briefly, fish were anesthetized by immersion in $0.033 \%$ aminobenzoic acid ethylmethylester (MS222; Sigma, St. Louis, MO) for $5 \mathrm{~min}$. A longitudinal incision was made at the side of the fish to expose the vertebral column. The spinal cord was cut completely between two vertebrae, $4 \mathrm{~mm}$ caudal to the brainstem-spinal cord junction.

L1.1 morpholino (5'-ATGAAAACAGCCCCGACTCCAGACA-3') and standard control morpholino (Gene Tools, Philomath, OR) were dissolved in Danieau solution (Nasevicius and Ekker, 2000) at different concentrations and were soaked onto small pieces of Gelfoam (Upjohn, Kalamazoo, MI) at a volume of $6 \mu \mathrm{l}$. These pieces of Gelfoam weredivided into 30 smaller pieces to yield 100-800 ng of morpholino per piece and were allowed to dry. Per fish, one of these pieces was applied to the 

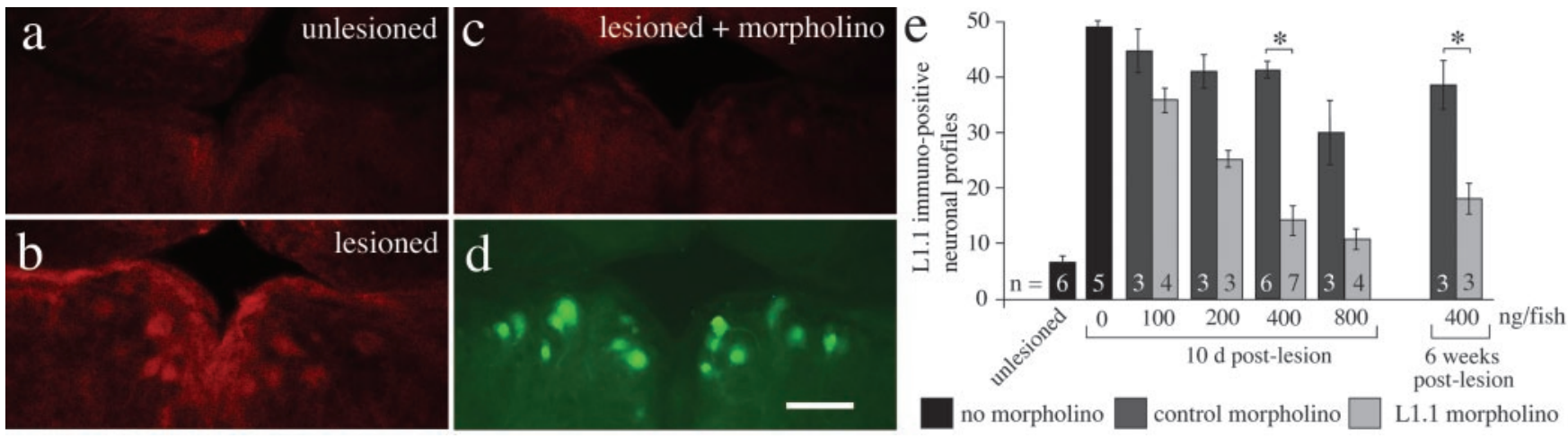

Figure 1. Morpholinos are retrogradely transported into neuronal somata in the brainstem and reduce L1.1 immunoreactivity. $a-d$, In a cross section through the NMLF, the number of L1.1-immunopositive neurons is increased $10 \mathrm{~d}$ after a lesion $(b)$ compared with unlesioned controls ( $a$ ). Upregulation is greatly reduced when L1.1 morpholino is applied to the spinal lesion site ( $c$ ). In the same tissue section $(d)$, accumulation of fluorescein-tagged L1.1 morpholino is visible with the appropriate filters. $e$, The number of L1.1-immunoreactive cell profiles is reduced by L1.1 morpholino compared with control morpholino-treated animals in a concentration-dependent manner $\left({ }^{*} p<0.05\right)$. Scale bar: (in $\left.d\right) a-d, 50 \mu \mathrm{m}$. Error bars represent SEM.

spinal transection site immediately after the transection. The wound was sealed with Histoacryl (B. Braun, Melsungen, Germany), and animals were allowed to survive the operation for up to 6 weeks. We used fluorescein-tagged and untagged morpholinos, which did not differ in their activities.

In some fish, axon regrowth was blocked by inserting a piece of Teflon tape ( $\sim 400 \times 400 \mu \mathrm{m}$; RS, Bad Hersfeld, Germany) at the spinal lesion site. The absence of supraspinal neurons with regenerated spinal axons was confirmed by retrograde tracing from a position caudal to the transection site after the recovery period and behavioral testing for locomotor activity at 6 weeks postlesion. A sham lesion was performed by opening the epidermis and exposing the vertebral column, which was left intact. Subsequently, the wound was sealed with Histoacryl.

The neuronal tracers biocytin (Sigma) and rhodamine dextran (Sigma) were used as described previously (Becker et al., 1997). To quantify regenerative success of brainstem neurons, horseradish peroxidase (HRP) (Roche, Mannheim, Germany) was applied to one spinal hemisphere from a position $8 \mathrm{~mm}$ caudal to the brainstem-spinal cord junction, which is $4 \mathrm{~mm}$ caudal to the spinal transection site. HRP labeling was developed as described previously (Becker et al., 1997), and labeled neuronal profiles were counted in vibratome sections (50 $\mu \mathrm{m}$ thick) of the brainstem. Analyzing spinal sections at the tracer application site showed no labeling of longitudinal tracts on the contralateral, unlabeled side, indicating that the tracer application was confined to one spinal hemisphere. For profile counting, the experimenter was blinded to the experimental treatment of individual fish.

Video tracking of freely moving fish. Swimming capabilities of fish at different postlesion time points and after different treatments were analyzed in two trials of $5 \mathrm{~min}$ each (intertrial interval, $4 \mathrm{hr}$ ). In each trial, the fish was placed in a brightly illuminated (100 lux) glass tank $(50 \times 30 \times$ $30 \mathrm{~cm})$ filled with aquarium water $\left(5 \mathrm{~cm}\right.$ deep; $\left.25^{\circ} \mathrm{C}\right)$. Trials were recorded using a video camera above the tank. Swim paths were tracked with Ethovision (Noldus, Wageningen, The Netherlands) software. Five samples were taken per second, and $0.7 \mathrm{~cm}$ was the minimal distance detected as movement between two consecutive samples. The mean of the entire length of the swim paths ("total distance moved") of the two trials was used for graphical representation and statistical analysis. The experimenter was blinded to the treatment of individual fish before testing.

Immunohistochemistry. Immunohistochemistry with a polyclonal antiserum against bacterially expressed zebrafish L1.1 (Becker et al., 2004) was performed on free-floating vibratome sections (50 $\mu \mathrm{m}$ thick) of brainstem and spinal cord, as described previously (Becker et al., 2004). Briefly, the sections were incubated with the antiserum (1:3000) overnight, and antibody binding was detected with the appropriate secondary cyanine-3-coupled antibodies (Dianova, Hamburg, Germany). For quantification, L1.1-positive cell profiles were counted in the brainstem using the $40 \times$ objective of an Axiophot (Zeiss, Oberkochen, Germany) microscope. The relative fluorescence intensity of the diffuse immuno- reactivity in the spinal cord was measured on digital photomicrographs taken on the Axiophot microscope using ImageJ software (http://rsb. info.nih.gov/ij/), as described previously (Becker et al., 2003). The experimenter was blinded to the experimental treatments before counting cell profiles and taking photomicrographs of spinal cord sections.

Western blot analysis. One millimeter pieces of spinal cord directly caudal to the lesion site and $4 \mathrm{~mm}$ caudal to the lesion site were isolated and subjected to standard Western blotting techniques, as described previously (Becker et al., 2003). The entire homogenate from one piece of spinal tissue was used to load one gel pocket. Stripping the filters and reprobing them with an anti-tubulin antibody (Sigma) served as a loading control.

Electron microscopy. For electron microscopy of anterogradely labeled synapses, biocytin was applied to the brainstem-spinal cord junction, as described previously (Becker et al., 1997), and allowed to be transported within axons over the spinal lesion site. Labeling was developed in $50 \mu \mathrm{m}$ sections, as described previously (Becker and Becker, 2001). Subsequently, sections were embedded in plastic resin, cut ultrathin $(80 \mathrm{~nm})$, and analyzed on a Zeiss EM900 electron microscope according to standard procedures. Synapses were identified by their vesicle content and the presence of a postsynaptic density. Labeled synapses of supraspinal origin appeared much darker in the electron microscope than unlabeled synapses of intraspinal origin (see Fig. $4 e, f$ ). The mean of the total number of labeled synaptic profiles in the spinal gray matter of two to three ultrathin sections, each from a different $50 \mu \mathrm{m}$ section per animal at a distance of $6 \mathrm{~mm}$ from the brainstem-spinal cord junction, was determined and used for graphical representation. The experimenter was blinded to the treatment of individual fish before counting synaptic profiles.

Statistical analysis. Because data were not normally distributed and different groups had unequal variances, nonparametric statistical analyses were performed with the software GraphPad Prism 3.0 (GraphPad Software, San Diego, CA) or StatView (SAS Institute, Cary, NC). Two treatments were compared with the Mann-Whitney $U$ test or $\chi^{2}$ test. To test the effect of more than two treatments, the Kruskal-Wallis test followed by Dunn's multiple comparisons test was used. Repeated measures within the same subjects were tested with the Friedman test followed by Dunn's multiple comparisons test. All of the tests were two-tailed. Data are presented as mean \pm SEM

\section{Results}

To establish the use of morpholinos in adult zebrafish, we first tested whether morpholinos would be taken up by brainstem neurons through their cut axons at a spinal lesion site, similar to conventional neuronal tracers such as biocytin (Becker et al., 1998). Application of fluorescein-tagged morpholinos (Fig. 1d) or biocytin, soaked on a Gelfoam pledget immediately after 

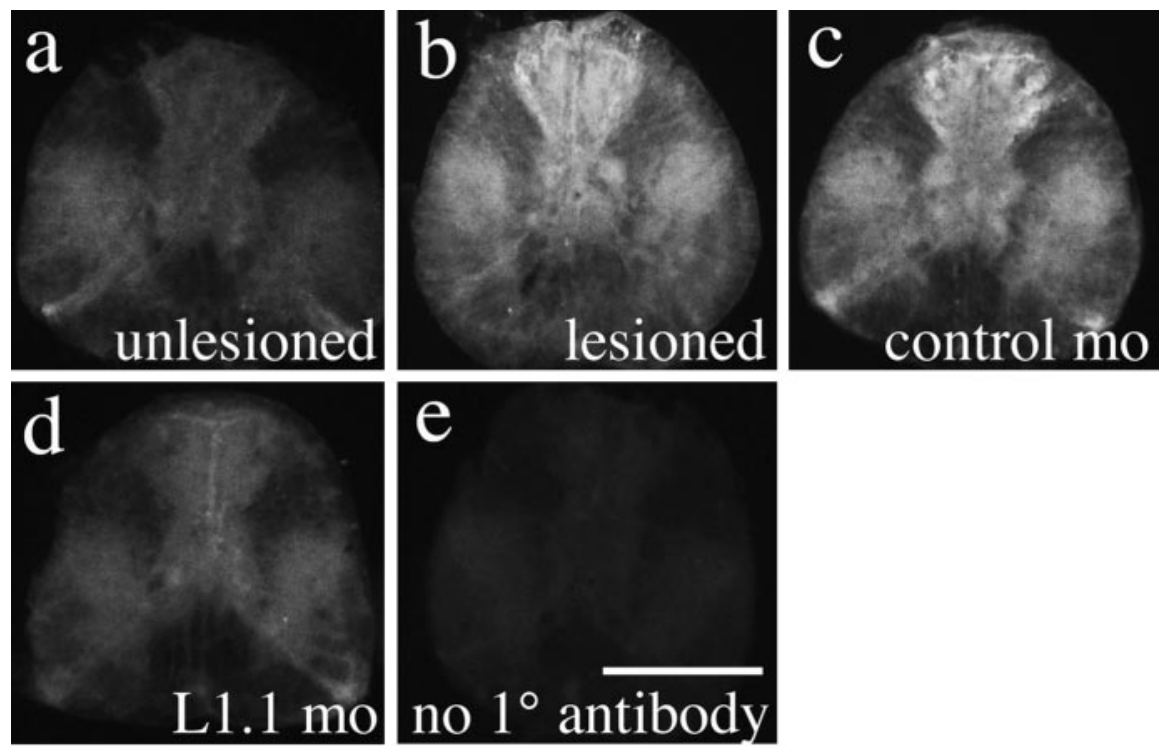

$\mathrm{f}$
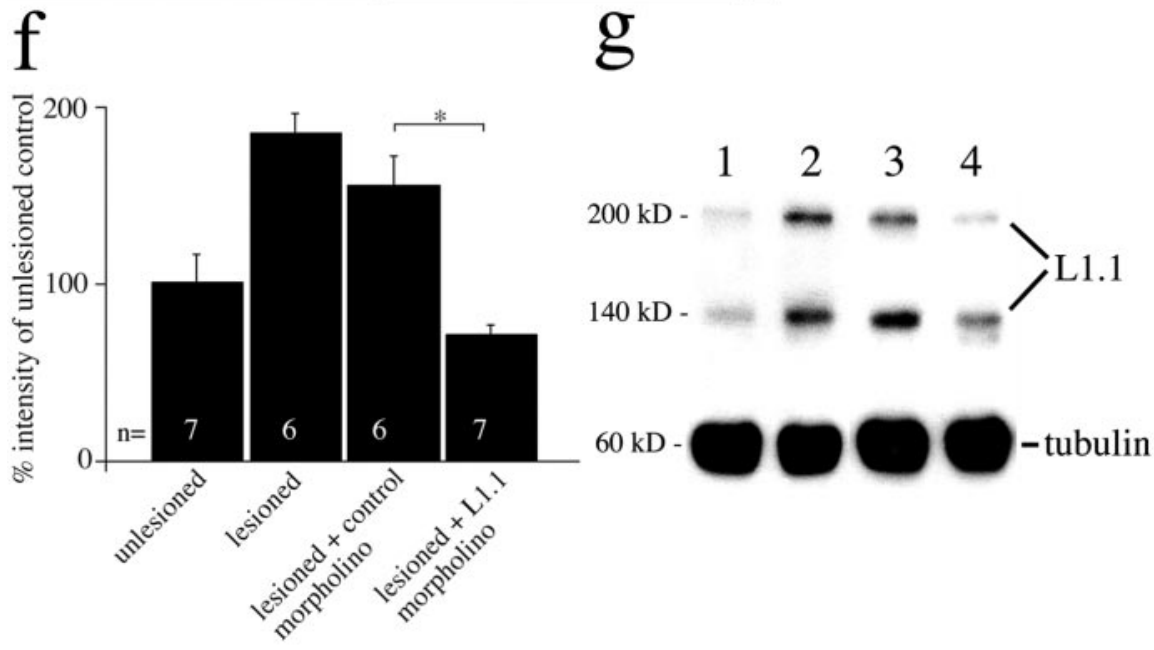

Figure 2. L1.1 morpholino reduces L1.1 immunolabeling caudal to a spinal transection site at $10 \mathrm{~d}$ postlesion. $a-e$, Immunofluorescence for $\mathrm{L} 1.1$ is increased $1 \mathrm{~mm}$ caudal to a spinal lesion site in lesioned animals $(b)$ and lesioned animals that received control morpholino $(c)$, but not in L1.1 morpholino (mo)-treated animals $(d)$, compared with unlesioned controls $(a)$ at $10 \mathrm{~d}$ postlesion. In e, the primary antibody $\left(1^{\circ}\right)$ was omitted on a spinal cross section from a lesioned animal. $f$, Quantification of $L 1.1$ immunofluorescence in spinal cross sections $1 \mathrm{~mm}$ caudal to the lesion site indicates a significant reduction by L1.1 morpholino treatment compared with control morpholino treatment $\left(^{*} p<0.05\right) . g$, Western blot analysis of the same spinal region indicates increased L1.1 immunoreactivity in a band at $200 \mathrm{kDa}$ and another band at $140 \mathrm{kDa}$, which probably represents a proteolytic degradation product of L1.1, in lesioned animals (lane 2) and those that had received the control morpholino (lane 3) compared with unlesioned controls (lane 1). Upregulation of L1.1 was weaker in L1.1 morpholino-treated animals (lane 4). Anti-tubulin labeling served as a loading control. Scale bar: (in e) $a-e, 100 \mu \mathrm{m}$. Error bars represent SEM.

spinal cord transection $4 \mathrm{~mm}$ caudal to the brainstem-spinal cord junction, did not show differences in the distribution and numbers of neuronal profiles specifically labeled in brainstem nuclei with spinal projections (data not shown). The fluorescein signal was still detectable in brainstem neurons 6 weeks after application. This indicates efficient retrograde transport of morpholinos.

To analyze whether a morpholino directed against L1.1 specifically reduces the expression of the protein, immunohistochemistry for L1.1 was performed on cross sections of brainstem and spinal cord after application of different morpholinos. In lesioned animals that did not receive morpholinos, the number of L1.1-immunopositive cell profiles was strongly increased in brainstem nuclei with spinal projections (Fig. $1 a, b, e$ ), confirming previous observations on L1.1
mRNA regulation (Becker et al., 1998). L1.1-specific morpholino, but not a control morpholino of a random sequence, reduced the number of L1.1-immunopositive cell profiles [e.g., in the anatomically well defined nucleus of the medial longitudinal fascicle (NMLF)] by maximally 66\% (at $400 \mathrm{ng}$ per animal) in a dose-dependent manner at $10 \mathrm{~d}$ postlesion (Kruskal-Wallis test followed by Dunn's test, $p<0.05$ for 400 ng per animal; $n \geq 3$ animals per concentration). Testing the efficient concentration at 6 weeks postlesion still indicated a significant reduction compared with control morpholino-treated animals (MannWhitney $U$ test, $p<0.05 ; n=3$ animals per treatment) (Fig. $1 b, c, e)$.

L1.1 immunoreactivity was also increased in the spinal gray matter after spinal cord transection within $1 \mathrm{~mm}$ of the lesion site (Fig. 2a,b,f), probably attributable to the presence of distal segments of regenerating descending axons and of spinal intrinsic neurons, which also upregulated L1.1 mRNA expression after spinal lesion (our unpublished observations). L1.1 morpholino reduced this immunoreactivity within $1 \mathrm{~mm}$ caudal to the lesion site, as indicated by a reduction of relative immunofluorescence in cross sections of the lesioned spinal cord (Fig. $2 c, d, f$ ) at $10 \mathrm{~d}$ postlesion (Kruskal-Wallis test followed by Dunn's test, $p<0.05$ for L1.1 morpholino vs control morpholino; $n \geq 6$ animals). Immunofluorescence within $1 \mathrm{~mm}$ of the lesion site was still reduced at 6 weeks postlesion. In Western blot analysis, labeling intensity of L1.1 was increased in lesioned animals and in lesioned animals that had received control morpholino. In those animals that received L1.1 morpholino, labeling intensity was always lower than in control morpholino-treated animals ( $n=3$ animals per treatment) (Fig. $2 g$ ). Four millimeters caudal to the lesion site, L1.1 immunoreactivity was more weakly increased after the lesion and was not significantly reduced after treatment with L1.1 morpholino, as detected by immunofluorescence and Western blot analysis (data not shown) at $10 \mathrm{~d}$ postlesion. The lack of a significant reduction of L1.1 immunolabeling at the greater distance may be explained by the observation that fewer neurons had taken up the morpholino at $4 \mathrm{~mm}$ from the lesion site. Application of fluorescently labeled morpholino indicated approximately four times fewer neurons that took up the morpholino per spinal cross section $4 \mathrm{~mm}$ caudal to the application site than $1 \mathrm{~mm}$ caudal to it (data not shown). Thus, L1.1 morpholino reduces L1.1 protein expression in brainstem neurons with spinal axons and near the spinal lesion site for at least 6 weeks postlesion.

To exclude a possible toxic effect of the morpholinos on cell survival, brainstem neurons were retrogradely traced by application of rhodamine dextran to the transection site together with 
L1.1-specific morpholinos (400 ng per animal). For example, in the NMLF, neither control morpholino $(42.0 \pm 6.34$ cell profiles per fish; $n=5$ animals) nor L1.1 morpholino $(49.0 \pm 5.18 ; n=4)$ reduced the number of neurons compared with lesioned animals that only received the tracer $(37.0 \pm 11.52 ; n=4$; Mann-Whitney $U$ test, $p>0.05) 6$ weeks after spinal cord transection and morpholino application. Thus, neurons in the brainstem survive morpholino application for at least 6 weeks.

To analyze the consequences of the reduced expression of L1.1 on functional recovery, we established a quantitative test of locomotor activity. We measured the total distance moved of undisturbed fish during 5 min trial periods by automatic video tracking (see Materials and Methods) (Fig. 3 ). This test exploits the natural behavior of zebrafish to swim almost constantly. At 1 week after a spinal cord transection, total distance moved was strongly reduced to $5 \%(70 \pm 22 \mathrm{~cm} / 5 \mathrm{~min} ; n=16)$ of that seen in unlesioned fish $(1302 \pm 302 \mathrm{~cm} / 5$

$\min ; n=10$ ) and recovered within 6 weeks postlesion to $57 \%$ (747 $\pm 137 \mathrm{~cm} / 5 \mathrm{~min}$; Dunn's multiple comparisons test after Friedman test, $p<0.01)$. No additional improvement was observed at 10 weeks postlesion (data not shown). Fish in which a piece of Teflon tape in the lesion site prevented regrowth of axons beyond the lesion site showed a totaldistance-moved value of $10 \%$ of that in unlesioned fish at 6 weeks postlesion $(134 \pm 40 \mathrm{~cm} / 5 \mathrm{~min} ; n=6)$. This differed significantly from lesioned fish without the block (Mann-Whitney $U$ test, $p<0.05)$. Sham-lesioned fish, in which the muscle tissue but not the spinal cord was lesioned, were not strongly impaired in locomotion and did not show differences between 1 and 10 weeks postlesion ( 1 week postlesion, $913 \pm 137 \mathrm{~cm} / 5 \mathrm{~min} ; 10$ weeks postlesion, $898 \pm 178 \mathrm{~cm} / 5 \mathrm{~min} ; n=10$; Friedman test, $p>$ $0.05)$. Thus, total distance moved is a parameter that is dramatically reduced after a spinal lesion and recovers over time, but not when regeneration is mechanically blocked.

At 6 weeks postlesion, fish that had received the L1.1 morpholino displayed reduced total-distance-moved values in a dosedependent manner to maximally $22 \%$ ( $166 \pm 28.6 \mathrm{~cm} / 5 \mathrm{~min} ; 400$ ng per fish; $n \geq 11$ animals per treatment) of the values for lesioned fish that had received control morpholino (769 \pm 174 $\mathrm{cm} / 5$ min; Dunn's multiple comparison test after Kruskal-Wallis test, $p<0.05$ for $400 \mathrm{ng}$ per animal) (Fig. $4 a$ ). At all of the concentrations tested (200-800 ng per fish), values for control morpholino-treated animals were not significantly different from animals that were lesioned but did not receive morpholino (Kruskal-Wallis test, $p>0.05$ ). The reduced values after L1.1 morpholino treatment were not different from those found for fish in which axon regrowth was blocked with a piece of Teflon tape $(134 \pm 39.5 \mathrm{~cm} / 5 \mathrm{~min}$; Mann-Whitney $U$ test, $p>0.05)$. Thus, L1.1 morpholino specifically reduces locomotor recovery to an extent that is similar to that in fish in which regrowth of axons over the lesion site is mechanically blocked.

To find anatomical correlates of this locomotor effect, the somata of brainstem neurons that regrew an axon beyond the spinal transection site were retrogradely traced in the same fish that had been tested in the locomotor paradigm. Intraspinal neurons with descending axons rarely regenerated their descendingaxons $(<5 \%)$ (our unpublished observations) and are therefore not considered in this analysis. HRP was applied $4 \mathrm{~mm}$ caudal to the spinal transection site to one spinal hemisphere 6 weeks after the lesion (Fig. $4 b, c$ ). The total number of cell profiles filled with HRP in cross sections of the entire brainstem of animals that had only received a gelatin foam pledget was set to $100 \%$. In animals that had received L1.1 morpholino, the number of labeled cell profiles was significantly reduced in a dose-dependent manner (to $35 \pm 22.1 \%$ ) compared with control morpholino-treated animals (106 \pm $34.95 \%$; Dunn's test for multiple comparisons after KruskalWallis test, $p<0.05 ; 400 \mathrm{ng}$ per animal) (Fig. 4d). Variabilities (SEM) for different groups are relatively high as a result of interindividual differences in regenerative success in zebrafish (Becker et al., 1997), also reported in other systems (Davis et al., 1990; Bareyre et al., 2004). Regression analysis of all of the control and L1.1 morpholino-treated animals indicated a significant correlation between the number of neuronal profiles back-labeled in the brainstem and total distance moved ( $r=$ $0.61 ; p=0.001 ; n=153)$. Thus, a reduction of axon regrowth from brainstem neurons occurs at the same concentration at which total distance moved was significantly reduced, and the number of brainstem neurons with regenerated axons correlates with the recovery of locomotor function of individual fish.

The above results suggest a role of regenerated axons from brainstem neurons for locomotor recovery. To analyze whether these axons reform synapses in the spinal cord caudal to a lesion site and whether their number is reduced by L1.1 morpholino treatment, we performed anterograde tracing. To label only newly formed synapses of regrown axons of supraspinal origin in the spinal cord distal to a lesion site, biocytin was applied at the brainstem-spinal cord junction 6 weeks after spinal cord lesion and morpholino application (400 $\mathrm{ng} /$ fish), $4 \mathrm{~mm}$ caudal to the 
a
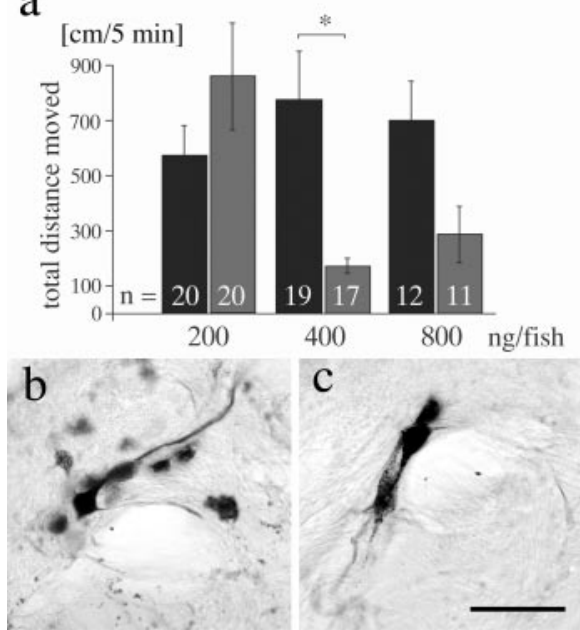

d

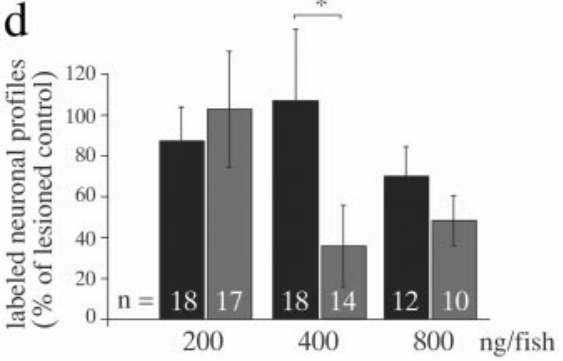

control morpholino $\square$ L1.1 morpholino

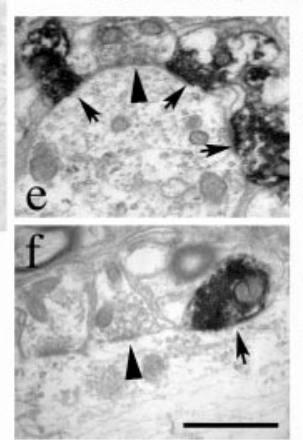

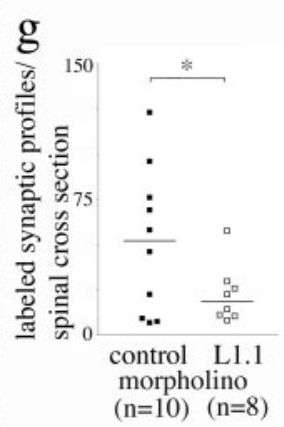

Figure 4. Morpholinos to L1.1 specifically impair locomotor and anatomical recovery 6 weeks after spinal cord transection. $a$, The total distance moved is reduced at $400 \mathrm{ng}$ of $\mathrm{L} 1.1$ morpholino per fish, compared with control morpholino $\left({ }^{*} p<0.05\right) . b, c$, Neuronal profiles in cross sections of the magnocellular octaval nucleus in the brainstem are back-labeled by HRP from a position caudal to the spinal transection site in control morpholino-treated $(b)$ and to a lesser extent in L1.1 morpholino-treated $(c)$ animals. Different focal planes are combined in the photomicrographs. $d$, The number of cell profiles back-labeled in the brainstem from a position caudal to a spinal transection site is significantly reduced after application of L1.1 morpholino compared with control morpholino at $400 \mathrm{ng}$ per fish. Values are expressed as percentage of the labeled profiles counted in lesioned animals that had not received morpholino $\left({ }^{*} p<0.05\right) . e-g$, The number of anterogradely traced synapses formed by regenerating axons from the brainstem caudal to a spinal transection site is reduced by L1.1 morpholino treatment. Labeled synapses of supraspinal origin (arrows) appear much darker than unlabeled synapses (arrowheads) of intraspinal neurons in the caudal spinal cord in control morpholino- $(e)$ and L1.1 morpholino-treated ( $f$ animals. In $g$, a graphic comparison of the numbers of labeled synapses found in individual animals is given (bars represent median values; ${ }^{*} p<0.05$ ). Scale bars: (in c) $b, c, 75 \mu \mathrm{m}$; (in $f$ ) $e, f, 1 \mu \mathrm{m}$. Error bars represent SEM.

brainstem-spinal cord junction. Anterogradely labeled synapses were analyzed in cross sections of the entire spinal cord $6 \mathrm{~mm}$ caudal to the brainstem-spinal cord junction, that is $2 \mathrm{~mm}$ caudal to the transection site (Fig. 4e,f). In unlesioned animals, $82.7 \pm$ 2.59 labeled synaptic profiles per section were found in this location ( $n=3$ animals). In lesioned animals that received control morpholino, the number of labeled synaptic profiles was lower $(53.3 \pm 12.76 ; 63 \%$ of the number seen in unlesioned controls; $n=10$ animals). This is compatible with the finding that not all brainstem neurons regenerate their spinal axons $(32-51 \%$ of all brainstem neurons compared with unlesioned controls), as reported previously (Becker et al., 1998). L1.1 morpholino treatment of lesioned animals reduced the number of labeled synaptic profiles to $22.3 \pm 5.75$ ( $n=8$ animals) (Fig. $4 g$ ), which is $43 \%$ of the number observed after treatment of lesioned animals with control morpholino ( $n=10$ animals; $\chi^{2}$ test, $\left.p<0.05\right)$. The reduction in synapse number after L1.1 morpholino treatment reflects the reduction in the number of brainstem neurons with regenerated spinal axons after L1.1 morpholino treatment to $35 \%$ compared with lesioned controls without morpholino application or control morpholino application (see above). Thus, brainstem neurons with spinal axons regrow synapses into the distal lesioned spinal cord, and their number is reduced after application of L1.1 morpholino.

\section{Discussion}

Our results indicate that the upregulation of L1.1 is an important part of the regenerative response of axotomized brainstem neurons in adult zebrafish. Reduced regrowth and synapse formation of regenerating axons from the brainstem caudal to a spinal lesion site may be one cause of the impaired functional recovery observed after L1.1 morpholino treatment. However, because L1.1 morpholino also accumulates in intraspinal neurons and has a measurable effect on L1.1 expression near the lesion site, the treatment could possibly also affect regeneration-related plasticity of intraspinal circuitry near the lesion site. An effect of the morpholino treatment on oligodendrocytes and astrocytes is unlikely because we could not detect L1.1 mRNA expression in putative oligodendrocytes in the white matter of the lesioned spinal cord (Becker et al., 1998), and double labeling with the astrocyte marker glial fibrillary acidic protein indicated no L1.1 mRNA expression in the somata of the radial astrocytes (data not shown). In addition, we describe a method to specifically reduce gene expression in axotomized neurons in an adult vertebrate for at least 6 weeks postlesion using antisense morpholino oligonucleotides. This method could also be useful in nonregenerating mammals to reduce expression of inhibitory molecules in the lesion site (Asher et al., 2001) or receptors for growth inhibitors in neurons (Lee et al., 2003), for example.

\section{References}

Asher RA, Morgenstern DA, Moon LD, Fawcett JW (2001) Chondroitin sulphate proteoglycans: inhibitory components of the glial scar. Prog Brain Res 132:611-619.

Bareyre FM, Kerschensteiner M, Raineteau O, Mettenleiter TC, Weinmann O, Schwab ME (2004) The injured spinal cord spontaneously forms a new intraspinal circuit in adult rats. Nat Neurosci 7:269-277.

Becker CG, Schweitzer J, Feldner J, Becker T, Schachner M (2003) Tenascin- $\mathrm{R}$ as a repellent guidance molecule for developing optic axons in zebrafish. J Neurosci 23:6232-6237.

Becker CG, Schweitzer J, Feldner J, Schachner M, Becker T (2004) Tenascin- $\mathrm{R}$ as a repellent guidance molecule for newly growing and regenerating optic axons in adult zebrafish. Mol Cell Neurosci 26: 376-389.

Becker T, Becker CG (2001) Regenerating descending axons preferentially reroute to the gray matter in the presence of a general macrophage/microglial reaction caudal to a spinal transection in adult zebrafish. J Comp Neurol 433:131-147.

Becker T, Wullimann MF, Becker CG, Bernhardt RR, Schachner M (1997) Axonal regrowth after spinal cord transection in adult zebrafish. J Comp Neurol 377:577-595.

Becker T, Bernhardt RR, Reinhard E, Wullimann MF, Tongiorgi E, Schachner M (1998) Readiness of zebrafish brain neurons to regenerate a spinal axon correlates with differential expression of specific cell recognition molecules. J Neurosci 18:5789-5803.

Bernhardt RR, Tongiorgi E, Anzini P, Schachner M (1996) Increased expression of specific recognition molecules by retinal ganglion cells and by 
the optic pathway glia accompanies the successful regeneration of retinal axons in adult zebrafish. J Comp Neurol 376:253-264.

Bulsara KR, Iskandar BJ, Villavicencio AT, Skene JH (2002) A new millenium for spinal cord regeneration: growth-associated genes. Spine 27:1946-1949.

Davis BM, Ayers JL, Koran L, Carlson J, Anderson MC, Simpson Jr SB (1990) Time course of salamander spinal cord regeneration and recovery of swimming: HRP retrograde pathway tracing and kinematic analysis. Exp Neurol 108:198-213.

Haspel J, Grumet M (2003) The L1CAM extracellular region: a multidomain protein with modular and cooperative binding modes. Front Biosci 8:s1210-s1225.

Heasman J (2002) Morpholino oligos: making sense of antisense? Dev Biol 243:209-214.

Lee DH, Strittmatter SM, Sah DW (2003) Targeting the Nogo receptorto treat central nervous system injuries. Nat Rev Drug Discov 2:872-878.

Martini R, Schachner M (1988) Immunoelectron microscopic localization of neural cell adhesion molecules (L1, N-CAM, and myelin-associated glycoprotein) in regenerating adult mouse sciatic nerve. J Cell Biol 106:1735-1746.
Nasevicius A, Ekker SC (2000) Effective targeted gene "knockdown" in zebrafish. Nat Genet 26:216-220.

Plunet W, Kwon BK, Tetzlaff W (2002) Promoting axonal regeneration in the central nervous system by enhancing the cell body response to axotomy. J Neurosci Res 68:1-6.

Roonprapunt C, Huang W, Grill R, Friedlander D, Grumet M, Chen S, Schachner M, Young W (2003) Soluble cell adhesion molecule L1-Fc promotes locomotor recovery in rats after spinal cord injury. J Neurotrauma 20:871-882.

Tongiorgi E, Bernhardt RR, Schachner M (1995) Zebrafish neurons express two L1-related molecules during early axonogenesis. J Neurosci Res 42:547-561.

Zhang Y, Campbell G, Anderson PN, Martini R, Schachner M, Lieberman AR (1995) Molecular basis of interactions between regenerating adult rat thalamic axons and Schwann cells in peripheral nerve grafts. 1. Neural cell adhesion molecules. J Comp Neurol 361:193-209.

Zhang Y, Roslan R, Lang D, Schachner M, Lieberman AR, Anderson PN (2000) Expression of CHL1 and L1 by neurons and glia following sciatic nerve and dorsal root injury. Mol Cell Neurosci 16: $71-86$. 